\title{
Multicenter inception cohort of enthesitis- related arthritis: variation in disease characteristics and treatment approaches
}

\author{
Sabrina Gmuca', Rui Xiao², Timothy G. Brandon' ${ }^{1}$, Ilaria Pagnini ${ }^{3}$, Tracey B. Wright ${ }^{4}$, Timothy Beukelman ${ }^{5}$,
} Esi M. Morgan ${ }^{6}$ and Pamela F. Weiss ${ }^{7,8^{*}}$

\begin{abstract}
Background: Enthesitis-related arthritis (ERA) is a specific subtype of juvenile idiopathic arthritis (JIA) defined according to the International League of Associations for Rheumatology (ILAR) criteria. We aimed to characterize the clinical features and treatment regimens in an inception cohort of children with ERA.

Methods: We performed a retrospective, cross-sectional, multicenter cohort study including subjects diagnosed with ERA between 1989 and 2012. Patients all fulfilled the ILAR criteria for ERA within 3 months of initial presentation to the rheumatology clinic. Differences in the prevalence of clinical criteria across study sites and by human leukocyte antigen (HLA)-B27 status were assessed using the Wilcoxon rank-sum or chi-square test, as appropriate.

Results: Two hundred thirty-four children met the inclusion criteria. Their median age at diagnosis was 11.6 years, and 59\% were HLA-B27-positive. Sixty-nine percent had enthesitis and arthritis at the time of diagnosis. Seventy-eight percent had a pauciarticular onset. The prevalence of all ILAR criteria at diagnosis, except arthritis and acute anterior uveitis, differed significantly across sites (all $p<0.01$ ). Medication use varied significantly across sites for children with peripheral arthritis $(p<0.001)$, but not for sacroiliitis or enthesitis only. Nonsteroidal anti-inflammatory drugs and disease-modifying antirheumatic drugs were the most commonly prescribed treatments, with anti-TNF agents primarily being initiation for sacroiliitis. HLA-B27 positivity was associated with male sex, higher active joint count, sacroiliitis, and higher disease activity at disease onset.

Conclusions: The majority of children had a pauciarticular onset, and several statistically significant clinical differences based on HLA-B27 status were identified. The observed heterogeneity in clinical presentation across sites reflects either true differences in patient populations or differences in how the ILAR criteria are being applied.
\end{abstract}

Keywords: Enthesitis-related arthritis, Juvenile idiopathic arthritis, HLA-B27, Classification criteria

\section{Background}

Juvenile idiopathic arthritis (JIA) is the most common pediatric rheumatic disease [1] and includes a number of heterogeneous arthritides. JIA is classified into seven mutually exclusive categories by the International League of Associations of Rheumatology (ILAR) classification [2]. Enthesitis-related arthritis (ERA) accounts

\footnotetext{
* Correspondence: weisspa@email.chop.edu

7University of Pennsylvania Perelman School of Medicine and Children's Hospital of Philadelphia, Philadelphia, PA, USA

${ }^{8}$ Department of Pediatric Rheumatology, The Children's Hospital of

Philadelphia, 34th Street and Civic Center Boulevard, Wood Building, Fourth

Floor, Philadelphia, PA 19104, USA

Full list of author information is available at the end of the article
}

for approximately $10 \%$ to $20 \%$ of JIA cases $[3,4]$ and, as alluded to by its name, is distinguished mainly by involvement of the entheses (where tendons and ligaments insert into bone). A significant proportion of current knowledge regarding ERA is based on data from adults with ankylosing spondylitis and spondyloarthritis (SpA), with a paucity of studies specifically focused on the ERA population. Additionally, application of adult data to children with ERA is not ideal, as evidenced by recognized clinical differences between the two populations, including less severe spinal involvement and more pronounced enthesitis and peripheral arthritis in children $[5,6]$. Therefore, there is a 
critical need for research focused specifically on the ERA population.

The majority of research conducted in children with ERA has been in the context of juvenile SpA, which includes other conditions such as inflammatory bowel disease and psoriatic arthritis. Epidemiologic studies focused on children with ERA as a distinct cohort are scarce. The largest study to date included 312 ERA subjects from a single center in northern India [7]. In this study, the mean age was 11.3 years, there was a male predominance $(9: 1)$, and $87 \%$ were human leukocyte antigen (HLA)-B27-positive. The high HLA-B27 positivity and single-center nature of the study make its applicability to other populations uncertain. Other studies focused on this JIA category have limited sample sizes ranging from 19 to 84 subjects [8-12]. Additionally, few studies have examined treatment practices for this group of children. Treatment recommendations have been published by the American College of Rheumatology (ACR) and are based primarily on the number of active joints and the presence of sacroiliitis [13]. It is unknown whether routine practice reflects these recommendations.

The significance of HLA-B27 positivity in this population has also not been well characterized. In children classified as having ERA on the basis of ILAR criteria, the proportion of HLA-B27 positivity ranges from $45 \%$ to $88 \%$ in published studies $[6,7,9,10,12]$. HLA-B27 positivity in this group of children also seems to vary by geographic location. Cohorts from India and Mexico have higher reported prevalence of HLA-B27 positivity than North American and European cohorts [7, 14]. The importance, or lack thereof, of HLA-B27 in shaping the clinical phenotype and course of these patients remains unclear. Both acute anterior uveitis (AAU) and sacroiliitis have been reported to have an association with HLA-B27 $[15,16]$. Researchers in prior studies have also reported HLA-B27 as an indicator of a poor prognosis among all categories of JIA, with HLA-B27-positive patients having more inflammatory back pain [17], greater disease activity [18], and decreased likelihood of remission after 8 years of disease [19] than children with JIA who are HLA-B27-negative.

We performed a retrospective, cross-sectional, multicenter cohort study that included subjects from five pediatric rheumatology centers (in North America and Europe) to evaluate the clinical presentation and treatment regimens of children with ERA. We also aimed to test whether children classified as having ERA are phenotypically distinct based on HLA-B27 positivity, which might have implications for the assessment or management of these two subgroups of ERA.

\section{Methods}

The protocol for this study was reviewed and approved by each institution's committee for the protection of human subjects.

\section{Study site and participants}

We performed a retrospective cross-sectional study using a multicenter database of children with ERA from five pediatric rheumatology centers. The study sites were Children's Hospital of Philadelphia (Philadelphia, PA, USA), Children's of Alabama (Birmingham, AL, USA), Cincinnati Children's Hospital Medical Center (Cincinnati, OH, USA), Texas Scottish Rite Hospital for Children (Dallas, TX, USA), and Meyer Children's Hospital (Florence, Italy). All subjects met the following inclusion criteria: (1) diagnosis of ERA according to the ILAR criteria (Table 1) within 3 months of initial presentation to rheumatology clinic with signs or symptoms of juvenile arthritis, (2) aged $\leq 16$ years at symptom onset, and (3) evaluated at one of the study sites during the study period (1989-2012). Chart reviewers independently applied the ILAR criteria to all study subjects to establish the diagnosis of ERA, given that this nomenclature did not exist for the years prior to 1995 . Eligibility criteria were verified by the coordinating center (Children's Hospital of Philadelphia); 85 children were excluded after this process.

\section{Clinical characteristics}

The baseline visit was defined as the first rheumatology appointment at which the patient exhibited clinical signs or symptoms of JIA (enthesitis, arthritis, AAU, or inflammatory back pain), regardless of whether the child was classified as having ERA at that time. Patient data were abstracted from the medical charts and included the following: demographics, family history, clinical features, patient-reported outcomes, and medications

Table 1 International League of Associations for Rheumatology criteria for enthesitis-related arthritis

\begin{tabular}{ll}
\hline Inclusion criteria & Exclusion criteria \\
\hline Arthritis and enthesitis & - Psoriasis or a history of psoriasis \\
& in the patient or a first degree \\
& relative \\
- Presence of IgM RF on at least & two occasions at least 3 months \\
or & apart \\
Arthritis or enthesitis with at least & - Systemic JIA in the patient
\end{tabular}

two of the following:

- Sacroiliac joint tenderness and/or

inflammatory spinal pain

- Presence of HLA-B27

- Onset of arthritis in a male over

6 years of age

- Family history in at least one first

degree relative of ankylosing

spondylitis, ERA, sacroiliitis with

$\mathrm{IBD}$, reactive arthritis or acute

anterior uveitis

- Acute anterior uveitis

Abbreviations: ERA Enthesitis-related arthritis, HLA Human leukocyte antigen, IBD Inflammatory bowel disease, IgM Immunoglobulin $M, R F$ Rheumatoid factor, JA Juvenile idiopathic arthritis 
prescribed. Peripheral arthritis was defined as joint swelling or, in the absence of swelling, limited range of motion plus tenderness [20, 21]. Enthesitis was defined as tenderness to palpation according to the ILAR criteria [2]. Because there is no standardized pediatric examination of the entheses, the sites routinely examined at each study site varied. The entheses insertion sites routinely examined included interosseous ligaments of the sacroiliac joint (all sites), hip flexors at the greater trochanter (four sites), quadriceps at the patella (all sites), infrapatellar tendon at the patella (all sites), infrapatellar tendon at the tibial tubercle (four sites), Achilles tendon (all sites), and plantar fascia at calcaneus (all sites). Sacroiliitis was defined as evidence of inflammation (e.g., bone marrow edema, joint space enhancement, or erosions/sclerosis) visualized by magnetic resonance imaging (MRI) [22, 23]. Ascertained laboratory values included the C-reactive protein (CRP), erythrocyte sedimentation rate (ESR), antinuclear antibody (ANA), and HLA-B27.

Patient-reported outcomes included patient-reported pain over the past week using a visual analogue scale (range 0-10); the Childhood Health Assessment Questionnaire (CHAQ), which is 30 -item questionnaire that assesses 8 domains of physical function with scores ranging from 0 to 3 [24, 25]; and patient global assessment of disease activity using a visual analogue scale (range 0 10). CHAQ scores of $0.13,0.63$, and 1.75 represent mild, mild to moderate, and moderate impairment, respectively [26]. Physician global assessment of disease activity (physician global) was also abstracted (range 0-10) [27, 28]. Two composite disease activity scores were calculated: the clinical Juvenile Arthritis Disease Activity Score (cJADAS) (comprised of the active joint count, physician global evaluation, and parent global evaluation) and the Juvenile Spondyloarthritis Disease Activity Index (JSpADA). The cJADAS was developed for use in all categories of JIA, with a score ranging from 0 to 30 , where higher scores indicate more active disease [29]. Cutoffs for minimal and high disease activity using the cJADAS are $<1.5$ and $>4$, respectively, in oligoarticular disease and $<2.5$ and $>8.5$, respectively, in polyarticular disease [30]. The JSpADA was developed specifically for use in juvenile SpA and consists of eight items (arthritis, enthesitis, patient pain assessment, inflammatory markers, morning stiffness, clinical sacroiliitis, AAU, and back mobility). Scores range from 0 to 8 , with higher scores being indicative of more active disease [31].

Because this study involved retrospective data collection, there were missing data. Missing data for laboratory values (ANA, HLA-B27, ESR, or CRP) did not exceed 13\%. Patient-reported outcomes were missing for approximately $50 \%$ of visits; missing values were not imputed. Age, sex, and active joint count did not significantly differ between those visits with and without missing patient-reported outcomes (all $p>0.05$ ) or between those with and without calculable cJADAS and JSpADA scores $(p>0.05)$.

For evaluation of treatments, children were considered to belong to one of the following three mutually exclusive phenotypes with the following hierarchy: (1) sacroiliitis (MRI-defined), (2) peripheral arthritis, and (3) enthesitis only. Treatments evaluated included nonsteroidal anti-inflammatory drugs (NSAIDs), intra-articular (IA) glucocorticoid injections, disease-modifying antirheumatic drugs (DMARDs: methotrexate, leflunomide, sulfasalazine), and biologic therapy with tumor necrosis factor (TNF)- $\alpha$ blockade (adalimumab, etanercept, infliximab). For comparison of clinical features between children based on HLA-B27 status, the cohort was restricted to those who had both arthritis and enthesitis. We reasoned that if we were to compare those who were HLA-B27-positive with those who were HLA-B27negative without restricting the analyses to those with both arthritis and enthesitis, we might be detecting differences based on the classification criteria met rather than true differences between these groups based on HLA-B27 status.

\section{Analysis}

Subject characteristics were summarized by median and interquartile range (IQR) for continuous variables and frequency and percentage for categorical variables. Differences in the prevalence of clinical criteria and medication use for clinical phenotypes across study sites were assessed by the chi-square test. Differences in clinical characteristics by HLA-B27 status were assessed using the Wilcoxon rank-sum or chi-square test, as appropriate. $P$ values less than 0.05 were considered statistically significant. All analyses were performed using Stata release 14.2 statistical software (StataCorp, College Station, TX, USA).

\section{Results}

\section{Clinical characteristics}

Two hundred thirty-four children and adolescents met the study inclusion criteria. Table 2 shows the demographic and clinical characteristics of this cohort at diagnosis. Children and adolescents with ERA were predominantly male (72.2\%) and Caucasian (82.9\%). The median age at diagnosis was 11.6 years (IQR 9.8-13.7). One hundred thirty-five (59.2\%) children were HLA-B27-positive. The median number of active joints was 2 (IQR 1-4), and the median number of tender entheses was 2 (IQR 1-4). The most commonly affected joints were the knee (107 patients with at least one affected knee [45.7\%]), ankle ( $n=$ 84 patients [35.9\%]), wrist $(n=46[19.7 \%])$, and hip $(n=45$ [19.2\%]). Seventy-eight percent of children had a 
Table 2 Patient characteristics at time of enthesitis-related arthritis classification

\begin{tabular}{ll}
\hline & $\begin{array}{l}\text { All subjects } \\
(n=234)\end{array}$ \\
\hline Demographics, median (IQR) or $n$ (\%) & $11.6(9.8-13.7)$ \\
Age in years & $169(72.2)$ \\
Sex, male & $194(82.9)$ \\
Race, Caucasian & $36(15.4)$ \\
Family history of HLA-B27-associated disease & \\
Clinical characteristics, median (IQR) or $n$ (\%) & $135(59.2)$ \\
HLA-B27 positivity & $2(1-4)$ \\
Active joint count & $2(1-4)$ \\
Tender entheses count & $25(55.6)$ \\
MRI-defined sacroiliitis ( $n=45$ with imaging) & $52(22.2)$ \\
Polyarticular onset & $49(23.4)$ \\
ANA-positive & $39(26.9)$ \\
CRP, abnormal/elevated & $93(45.8)$ \\
ESR, abnormal/elevated & \\
Patient-/physician-reported outcomes, median (IQR) & \\
Physician global disease activity assessment (0-10) & $2.5(2-4)$ \\
Juvenile Spondyloarthritis Disease Activity Index & $3(2.5-4)$ \\
(JSpADA) (0-8) & $9(5-14)$ \\
Juvenile Arthritis Disease Activity Score (cJADAS), \\
excluding ESR (0-30) \\
$\begin{array}{l}\text { Patient/parent global disease activity assessment } \\
\text { (0-10) }\end{array}$ \\
$\begin{array}{l}\text { Function (CHAQ) (0-3) } \\
\text { Patient/parent pain assessment (0-10) }\end{array}$ \\
\hline Abbrevatons IQR Int \\
$0.563(0-1)$ \\
$4(2-7)$ \\
\hline
\end{tabular}

Abbreviations: IQR Interquartile range, HLA Human leukocyte antigen, MRI Magnetic resonance imaging, ANA Antinuclear antibody, CRP C-reactive protein, ESR Erythrocyte sedimentation rate, CHAQ Childhood Health Assessment Questionnaire

Denominators for clinical characteristics varied because of missing data: HLAB27 status $(n=228)$, magnetic resonance imaging-defined sacroiliitis $(n=45)$, ANA $(n=209)$, CRP $(n=145)$, ESR $(n=203)$, physician global disease activity assessment $(n=126)$, JSpADA $(n=116)$, patient/parent global disease activity assessment $(n=102)$, CHAQ $(n=120)$, patient/parent pain assessment $(n=129)$, CJADAS (JADAS3-10) $(n=86)$

pauciarticular onset (fewer than five joints). The most frequently tender entheses were the insertions of the infrapatellar tendon on the patella ( $n=103$ patients [44\%]), Achilles tendon $(n=77$ [32.9\%]), and the interosseous ligaments of the sacroiliac joint $(n=71$ [30.3\%]).

Figure 1 shows the percentage of children who met each of the ILAR ERA criteria. The majority of patients met the ILAR criteria for a diagnosis of ERA at the first rheumatology visit $(n=214$ [91.5\%]), with 18 subjects (7.7\%) meeting the criteria at the second visit and 2 subjects $(0.9 \%)$ meeting the criteria by the third visit. One hundred sixty-two (69.2\%) children had arthritis and enthesitis, and 72 (30.8\%) children had arthritis or enthesitis plus at least two additional ILAR criteria. Of the 72 children presenting with arthritis or enthesitis plus at least two additional ILAR criteria, 55 had arthritis only and 17 had enthesitis only. Because arthritis (91.5\%) and enthesitis (75.2\%) are requirements under the ILAR criteria for ERA, it was not surprising that they were the two most common disease manifestations followed by the presence of arthritis in a male over the age of 6 years $(62.8 \%)$. Sacroiliac tenderness was present in $25.6 \%(n=60)$ of patients, and AAU was present in only $5.6 \%(n=13)$ of patients. Although more than half (59.2\%) of all patients were HLA-B27-positive, this proportion varied significantly across sites, ranging from $35 \%$ to $89 \%(p<0.001)$ (Fig. 1). The prevalence of arthritis and AAU did not differ significantly across sites. The prevalence of each of the remaining ILAR criteria, however, was also significantly different across sites (Fig. 1) (all $p<0.01$ ): enthesitis (range: $57 \%$ to 91\%), sacroiliac tenderness or inflammatory lumbosacral pain (range: $12 \%$ to $43 \%$ ), onset of arthritis in a male over 6 years (range: $46 \%$ to $79 \%$ ), and history of HLA-B27associated disease in a first-degree relative (range: $6 \%$ to $43 \%)$.

Table 2 lists patient- and physician-reported disease activity assessments. Disease activity at diagnosis was moderate as assessed globally by patients/parents (median 4, IQR 2-6) and physicians (median 2.5, IQR 2-4). Patients also had moderate disease activity as evaluated by composite disease activity measures (JSpADA median 3, IQR 2.5-4; cJADAS median 9, IQR 5-14). Patients reported moderate pain (median score of 4, IQR 2-7) and between mild and mild to moderate disability as assessed by the CHAQ (median score of 0.563 , IQR $0-1$ ). Twenty-seven percent $(n=39)$ had systemic inflammation as evidenced by an elevated CRP level, and $45.8 \%$ $(n=93)$ had an elevated ESR (Table 2).

\section{Medication use}

Figure 2 demonstrates unadjusted medication use at diagnosis based on clinical phenotype. Overall, NSAIDs were the most commonly prescribed medication class $(n$ $=189[80.8 \%])$, followed by DMARDs $(n=75$ [32.1\%]). Oral glucocorticoids (14.6\%), IA glucocorticoid injections (15.0\%), and biologic agents (15.4\%) were less commonly used. Medication use varied significantly among sites for children with peripheral arthritis $(p<0.001)$ but not for those with sacroiliitis or enthesitis only. NSAIDs-only was the most prevalent treatment across every disease phenotype. Children presenting with MRIdefined sacroiliitis were prescribed NSAIDs only (40\%), biologic monotherapy (32\%), or a combination of a biologic and a DMARD (4\%). Children with arthritis at diagnosis were most commonly prescribed NSAIDs only (43\%) or DMARD monotherapy (27\%). Subjects with only enthesitis at diagnosis were treated primarily with NSAIDs (82\%). IA glucocorticoid injections were 


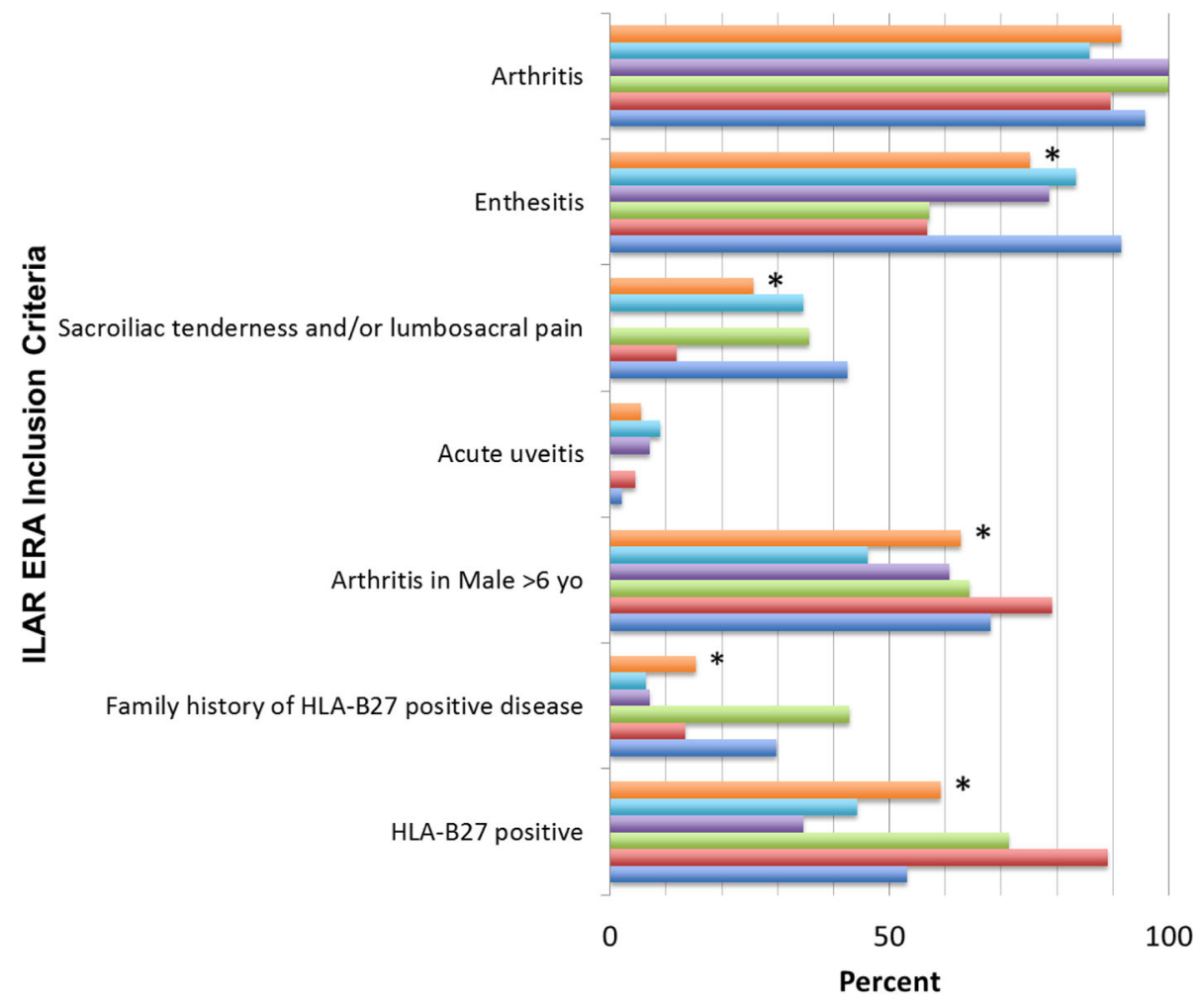

ALL Site 1 Site $2 \quad$ Site $3 \quad$ Site $4 \quad$ Site 5

Fig. 1 International League of Associations for Rheumatology (ILAR) enthesitis-related arthritis (ERA) inclusion criteria by enrollment site. ${ }^{*} p<0.05$ for between-group comparisons. HLA Human leukocyte antigen

reserved for children with peripheral arthritis (11.3\%). Oral corticosteroids were prescribed for those with sacroiliitis (4.0\%) and peripheral arthritis (5.9\%).

\section{HLA-B27 status and clinical phenotype}

In order to determine whether patient demographics, clinical characteristics, or ILAR criteria fulfillment differed on the basis of HLA-B27 status, we restricted our analyses to patients who met ILAR criteria by having both arthritis and enthesitis $(n=156)$. Clinical characteristics of HLA-B27-positive versus HLA-B27-negative children are presented in Table 3. Among this restricted cohort, $44 \%(n=69)$ were HLA-B27-positive. As in the primary analysis, male sex and Caucasian racial predominance persisted.

There was an older median age of onset in the HLAB27-positive children (12.3 versus 11.0 years; $p=0.01$ ). In comparison with HLA-B27-negative children, HLAB27-positive children had a higher active joint count $(p$ $<0.01)$ and were more likely to have sacroiliitis $(p<$ 0.01). A greater proportion of HLA-B27-positive children had an elevated ESR (54.8\% versus $31.1 \% ; p<0.01$ ). The JSpADA was significantly higher in those who were HLA-B27-positive than in those who were HLA-B27- negative $(p=0.04)$, whereas there was no significant difference in the cJADAS or physician global disease activity assessment. Those who were HLA-B27-positive were more likely to develop arthritis after the age of 6 years $(p<0.01)$. There were no other differences in the ILAR criteria based on HLA-B27 status.

\section{Discussion}

To our knowledge, this study comprises the largest multicenter international inception cohort of children with ERA to date. Children with ERA were predominantly male, Caucasian, and HLA-B27-positive; these findings are in accordance with previously published cohorts $[9,32]$. An overwhelming majority of patients (91.5\%) met the ILAR criteria for a diagnosis of ERA at their first visit to a pediatric rheumatologist. The majority of subjects had both arthritis and enthesitis at the time of diagnosis. Most children had a pauciarticular onset of disease, and the most commonly affected joints were the knees, ankles, wrists, and hips. Enthesitis was most common at the attachments of the infrapatellar tendon on the inferior pole of the patella, the interosseous ligaments of the sacroiliac joints, and the Achilles tendon. These findings highlight the importance of carefully assessing for arthritis 


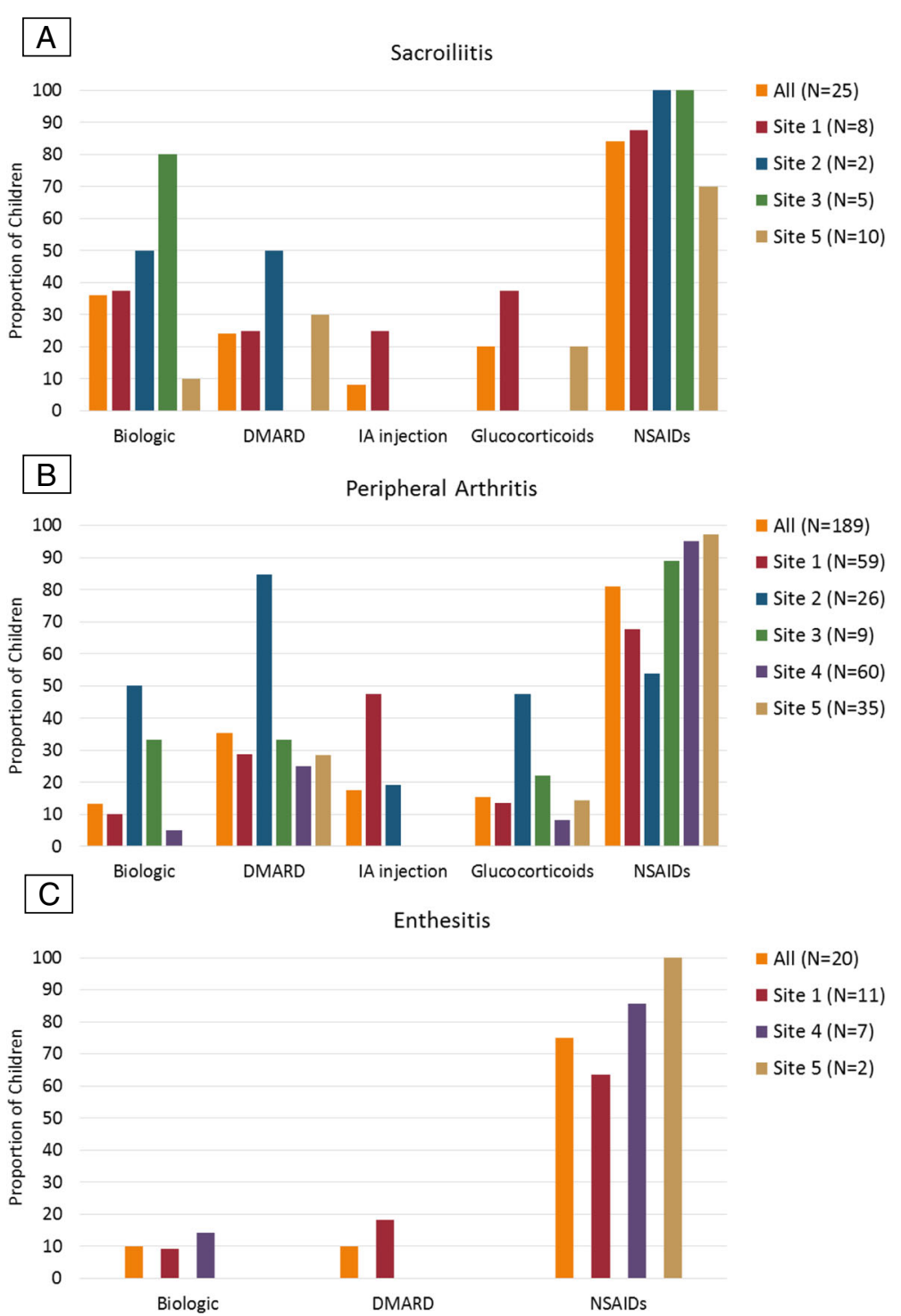

Fig. 2 Medications prescribed based on clinical phenotype. Medications prescribed across sites for (a) sacroiliitis (magnetic resonance imaging [MRI]-defined), (b) peripheral arthritis, and (c) enthesitis only. Children were considered as having one of the following three mutually exclusive phenotypes with the following hierarchy: (1) sacroiliitis (MRI-defined), (2) peripheral arthritis, and (3) enthesitis. Biologic medications included tumor necrosis factor-a inhibitors (adalimumab, etanercept, infliximab). Sacroiliitis data were not available for site 4 . No children from site 2 or 3 were diagnosed with isolated enthesitis, and of those with enthesitis, none were prescribed IA injections or glucocorticoids. DMARD Disease-modifying antirheumatic drug, Glucocorticoids Oral glucocorticoids, IA Intra-articular glucocorticoid injection, NSAIDs Nonsteroidal anti-inflammatory drugs

and enthesitis in all possible locations when examining a new patient presenting with concern for JIA. Perhaps special attention should be paid to the aforementioned anatomical sites when considering a diagnosis of ERA. NSAIDs and DMARDs were the most often prescribed first-line therapies. There was increased use of biologic agents, however, for the treatment of children with sacroiliitis. Systemic glucocorticoids were reserved for children with arthritis or sacroiliitis. Children who were HLA-B27positive tended to be older and male, with a higher active joint count, more sacroiliitis, and higher disease activity.
Several findings warrant additional discussion. First, we found significant variability in the presenting features and initial treatment strategies between sites. The variation in presenting features may reflect either true differences by geographic location or differences between sites in the assessment of particular features. There are no standardized definitions for what constitutes positive enthesitis. The entheses examined and thresholds for declaring positive findings varied not only between sites but also between physicians at each site. Variation in family history could reflect recall bias, differences in 
Table 3 Clinical features by human leukocyte antigen B27 status

\begin{tabular}{|c|c|c|c|c|}
\hline & $\mathrm{All}^{\mathrm{a}}(n=156)$ & HLA-B27 positive $(n=69)$ & HLA-B27 negative $(n=87)$ & $p$ Value \\
\hline \multicolumn{5}{|l|}{ Demographics and clinical findings, $n(\%)$ or median (IQR) } \\
\hline Age in years & $11.5(9.6,13.6)$ & $12.3(10.3,14.1)$ & $11.0(8.8,13.2)$ & $<0.01$ \\
\hline Sex, male & $100(64.1)$ & $50(72.5)$ & $50(57.5)$ & 0.05 \\
\hline Race, Caucasian & $129(82.7)$ & $59(85.5)$ & $70(80.5)$ & 0.66 \\
\hline Active joint count & $2(1-4)$ & $3(2-5)$ & $2(1-4)$ & $<0.01$ \\
\hline Tender entheses count & $2(2-4)$ & $2(1-3)$ & $2(2-5)$ & 0.01 \\
\hline MRI-defined sacroiliitis & $22(57.9)$ & $15(83.3)$ & $7(35.0)$ & $<0.01$ \\
\hline Polyarticular onset & $36(23.1)$ & $20(29.0)$ & $16(18.4)$ & 0.15 \\
\hline ANA-positive & $31(21.4)$ & $10(15.6)$ & $21(25.9)$ & 0.13 \\
\hline CRP, abnormal/elevated & $31(28.4)$ & $13(31.0)$ & $18(26.9)$ & 0.65 \\
\hline ESR, abnormal/elevated & $57(41.9)$ & $34(54.8)$ & $23(31.1)$ & $<0.01$ \\
\hline \multicolumn{5}{|l|}{ Outcome measures, median (IQR) } \\
\hline Physician disease activity (0-10) & $2.4(2-3)$ & $2(2-3)$ & $2.6(2-4)$ & 0.07 \\
\hline Juvenile Spondyloarthritis Disease Activity Index (JSpADA) (0-8) & $3(2.5-4)$ & $3.5(2.5-4)$ & $3(2.5-3.5)$ & 0.04 \\
\hline Patient/parent disease activity (0-10) & $4(2-6)$ & $4(2-5)$ & $4(2-6)$ & 0.63 \\
\hline Function (CHAQ) (0-3) & $0.625(0-1)$ & $0.375(0-1)$ & $0.625(0-1)$ & 0.85 \\
\hline Patient/parent pain (0-10) & $4(2-7)$ & $4(3-7)$ & $4(2-7)$ & 0.71 \\
\hline $\begin{array}{l}\text { Juvenile Arthritis Disease Activity Score, excluding ESR (cJADAS) } \\
(0-30)\end{array}$ & $9(5-13)$ & $9(5-13)$ & $9(5-14)$ & 0.98 \\
\hline \multicolumn{5}{|l|}{ ILAR criteria, $n(\%)$} \\
\hline Acute, symptomatic uveitis & $7(4.5)$ & $4(5.8)$ & $3(3.5)$ & 0.48 \\
\hline Arthritis in a male over 6 years of age & $93(59.6)$ & $50(72.5)$ & $43(49.4)$ & $<0.01$ \\
\hline Family history of HLA-B27-associated disease & $18(11.5)$ & $7(10.1)$ & $11(12.6)$ & 0.63 \\
\hline Sacroiliac tenderness or lumbosacral pain & $46(29.5)$ & $20(29.0)$ & $26(29.9)$ & 0.9 \\
\hline
\end{tabular}

Abbreviations: HLA-B27 Human leukocyte antigen B27, MRI Magnetic resonance imaging, ANA Antinuclear antibody, CRP C-reactive protein, ESR Erythrocyte sedimentation rate, IQR Interquartile range, CHAQ Childhood Health Assessment Questionnaire

Analyses restricted to patients who had both arthritis and enthesitis at the time of diagnosis. Denominators varied due to missing data: MRI-defined sacroiliitis $(n=38) ;$ ANA ( $n=145) ;$ CRP $(n=109)$; ESR $(n=136)$; Physician disease activity $(n=97) ;$ JSpADA $(n=92) ;$ Patient/parent disease activity $(n=79) ;$ CHAQ $(n=92)$; Patient/parent pain $(n=100)$; cJADAS (JADAS3-10) $(n=69)$

${ }^{\text {a }}$ Six subjects who had arthritis and enthesitis at diagnosis were excluded because they were missing HLA-B27 status. P-values less than 0.05 are in italics and considered clinically significant

how family history was recorded at each site, or true variation. In terms of the initial treatment of ERA, we found that NSAIDs and DMARDs were most often prescribed. We did note, however, that there was increased use of biologic agents for the treatment of children with sacroiliitis, and IA glucocorticoids were reserved for cases of peripheral arthritis. Despite the intersite variation in treatments, our findings suggest that pediatric rheumatologists are generally adhering to ACR-recommended treatment guidelines $[13,33,34]$ to initiate a TNF- $\alpha$ blocker for axial disease, especially early in the disease course [35]; however, barriers imposed by insurance companies may account for the fact that only $15 \%$ of patients received biologic agents in the first 3 months of disease. Because many insurance companies require that patients first fail methotrexate before they will provide coverage for a biologic agent, this logistical issue may be driving our findings. These differences highlight the need for standardized assessments for ERA as well as comparative effectiveness studies of the different treatment options.

Second, this study demonstrates a few statistically significant clinical differences based on HLA-B27 status. Children who were HLA-B27-positive tended to be older and male, with a higher active joint count and more sacroiliitis. We restricted our analyses of HLA-B27positive versus HLA-B27-negative patients to children with both arthritis and enthesitis because of a concern for selection bias owing to the inherent nature of the minor inclusion classification criteria. Doing so strengthened our findings by removing any reliance on criteria selecting for HLA-B27 status, sex, and age. HLA-B27positive children also had a higher disease activity as assessed by the JSpADA. Surprisingly, in contrast to adult studies, we did not find an association between HLA-B27 status and the presence of AAU [15, 36]. However, in a recent study of patients with ERA in 
which researchers enrolled subjects prospectively over a 3 -year period an overall prevalence of AAU of 15.4\% was found with no statistically significant difference based on HLA-B27 status [7]. This suggests that there may be additional phenotypic differences between child- and adult-onset diseases. Alternatively, one might theorize that AAU manifests later in the disease course of ERA and is not a frequent symptom at the time of initial presentation. Because this was a cross-sectional study at the time of diagnosis, it is possible that a larger proportion of patients eventually developed AAU. Additionally, we did not find any statistically significant difference in HLA-B27-positive and HLA-B27-negative children with regard to a family history of HLA-B27-associated disease, which challenges current understanding of the genetic susceptibility of patients to ERA. One possibility is that adult-onset SpA is more greatly affected by one's genetic milieu and therefore is more common among related family members. Perhaps the development of ERA is influenced less by genetics but more so by environmental factors. Alternatively, given that family history was based on patients' individual reports, it could be that patients' knowledge of HLA-B27associated diseases in their own family history may be limited. Longitudinal studies should further assess the significance of HLA-B27 as a prognostic marker over the natural history of ERA and whether enthesitis follows a different pattern in HLA-B27-negative versus HLA-B27-positive patients.

Our findings should be interpreted in light of several limitations. First, this was a retrospective study spanning several decades from the earliest to the most recent patient. As such, there was not uniform collection of data at the time of initial evaluation, and clinical examinations may have varied between providers and sites. We suspect that treatment practices did vary over time with likely increasing use of TNF$\alpha$ blockade, but this type of time-series analysis would be best suited as its own separate study. Second, imaging was done at the discretion of the caregiver; there was no uniform screening for hip arthritis or sacroiliitis with imaging. Several reports have shown that sacroiliitis (with sclerotic changes and erosions) is frequently present in children who do not complain of back pain $[16,37,38]$. Therefore, the prevalence of MRI-confirmed sacroiliitis in both groups in this cohort was likely underreported, and our results are likely biased toward the null.

To our knowledge, this study is the largest multicenter international study of children with ERA to date. Both the presenting clinical features and treatment algorithms used for various phenotypes of ERA significantly differed across sites. This heterogeneity in clinical presentation reflects either true differences in patient populations or differences in how the ILAR criteria are being applied. The variation in treatment algorithms emphasizes the need for efficacy and effectiveness studies to help establish evidence-based treatment guidelines tailored to the various clinical phenotypes in this understudied population.

\section{Conclusions}

To our knowledge, this study comprises the largest multicenter international inception cohort of children with ERA to date. Both the presenting clinical features and treatment algorithms used for the various phenotypes of ERA differed significantly across clinical sites. NSAIDs and DMARDs were prescribed as first-line treatments for newly diagnosed ERA, with biologic agents used primarily for sacroiliitis. In comparison with children who were HLA-B27-negative, children who were HLA-B27positive tended to be older and male and had a higher active joint count, more sacroiliitis, and greater disease activity.

\section{Abbreviations}

AAU: Acute anterior uveitis; ACR: American College of Rheumatology; ANA: Antinuclear antibody; CHAQ: Childhood Health Assessment

Questionnaire; CJADAS: clinical Juvenile Arthritis Disease Activity Score; CRP: C-reactive protein; DMARD: Disease-modifying antirheumatic drug; ERA: Enthesitis-related arthritis; ESR: Erythrocyte sedimentation rate; HLA: Human leukocyte antigen; IA: Intra-articular; IBD: Inflammatory bowel disease; IgM: Immunoglobulin M; ILAR: International League of Associations for Rheumatology; IQR: Interquartile range; JIA: Juvenile idiopathic arthritis; JSpADA: Juvenile Spondyloarthritis Disease Activity Index; MRI: Magnetic resonance imaging; NSAID: Nonsteroidal anti-inflammatory drug;

RF: Rheumatoid factor; SpA: Spondyloarthritis; TNF-a: Tumor necrosis factor-a

\section{Acknowledgements}

The authors thank Colden Broderick, Andrew Klink, Keshia Maughn, Christy Reed, Davide Moretti, Roxanna Sabghi, and Carla Upperman for their assistance in review of the medical charts and data entry.

\section{Funding}

Research reported in this publication was supported by the National Institute of Arthritis and Musculoskeletal and Skin Diseases of the National Institutes of Health under award numbers T32-AR007442 (to SG) and K23-AR059749 (to PFW). The content is solely the responsibility of the authors and does not necessarily represent the official views of the National Institutes of Health.

\section{Availability of data and materials}

The datasets used and/or analyzed during the present study are available from the corresponding author on reasonable request.

\section{Authors' contributions}

PFW was responsible for study conception and design. EMM, IP, TB, TBW $T G B$, and PFW were responsible for acquisition of the data. PFW, RX, SG, and TGB participated in interpretation and analysis of the data. PFW, RX, SG, and TGB drafted and wrote the manuscript. EMM, IP, TB, and TBW were involved in critically revising the manuscript for important intellectual content. All authors read and approved the final manuscript and take accountability for the accuracy and integrity of the final version.

Competing interests

The authors declare that they have no competing interests.

Consent for publication

Not applicable. 


\section{Ethics approval and consent to participate}

Because this was a multicenter study, each institution's committee for the protection of human subjects approved this study, with the institutional review board (IRB) at The Children's Hospital of Philadelphia serving as the governing IRB. These institutions include the University of Florence and Anna Meyer Children's Hospital, Florence, Italy; the University of Texas Southwestern Medical Center and Texas Scottish Rite Hospital of Children, Dallas, TX, USA; the University of Alabama at Birmingham, Birmingham, AL, USA; and Cincinnati Children's Hospital, Cincinnati, OH, USA. Because this was a retrospective study, a waiver of assent/consent was obtained.

\section{Publisher's Note}

Springer Nature remains neutral with regard to jurisdictional claims in published maps and institutional affiliations.

\section{Author details}

${ }^{1}$ Division of Rheumatology, Department of Pediatrics, The Children's Hospital of Philadelphia, Philadelphia, PA, USA. ${ }^{2}$ Department of Biostatistics and Epidemiology, Perelman School of Medicine, University of Pennsylvania, Philadelphia, PA, USA. ${ }^{3}$ University of Florence and Anna Meyer Children's Hospital, Florence, Italy. ${ }^{4}$ University of Texas Southwestern Medical Center and Texas Scottish Rite Hospital of Children, Dallas, TX, USA. ${ }^{5}$ University of Alabama at Birmingham, Birmingham, AL, USA. ${ }^{6}$ Cincinnati Children's Hospital, Cincinnati, OH, USA. 7 University of Pennsylvania Perelman School of Medicine and Children's Hospital of Philadelphia, Philadelphia, PA, USA. ${ }^{8}$ Department of Pediatric Rheumatology, The Children's Hospital of Philadelphia, 34th Street and Civic Center Boulevard, Wood Building, Fourth Floor, Philadelphia, PA 19104, USA.

Received: 23 January 2017 Accepted: 7 April 2017

Published online: 02 May 2017

\section{References}

1. Ravelli A, Martini A. Juvenile idiopathic arthritis. Lancet. 2007;369:767-78.

2. Petty RE, Southwood TR, Manners P, Baum J, Glass DN, Goldenberg J, et al. International League of Associations for Rheumatology classification of juvenile idiopathic arthritis: second revision, Edmonton, 2001. J Rheumatol. 2004;31:390-2.

3. Flato B, Hoffmann-Vold AM, Reiff A, Forre O, Lien G, Vinje O. Long-term outcome and prognostic factors in enthesitis-related arthritis: a case-control study. Arthritis Rheum. 2006;54:3573-82.

4. Bryan AR, Rabinovich CE. Enthesitis-related arthritis: time to re-define? Curr Rheumatol Rep. 2014;16:466

5. Hofer M. Spondylarthropathies in children-are they different from those in adults? Best Pract Res Clin Rheumatol. 2006;20:315-28.

6. Adrovic A, Barut K, Sahin S, Kasapcopur O. Juvenile spondyloarthropathies. Curr Rheumatol Rep. 2016;18:55.

7. Srivastava R, Phatak S, Yadav A, Bajpai P, Aggarwal A. HLA B27 typing in 511 children with juvenile idiopathic arthritis from India. Rheumatol Int. 2016;36: 1407-11.

8. Weiss PF, Klink AJ, Behrens EM, Sherry DD, Finkel TH, Feudtner C, et al. Enthesitis in an inception cohort of enthesitis-related arthritis. Arthritis Care Res (Hoboken). 2011;63:1307-12.

9. Nordal E, Zak M, Aalto K, Berntson L, Fasth A, Herlin T, et al. Ongoing disease activity and changing categories in a long-term Nordic cohort study of juvenile idiopathic arthritis. Arthritis Rheum. 2011;63:2809-18.

10. Thomson W, Barrett JH, Donn R, Pepper L, Kennedy L, Ollier WE, et al. Juvenile idiopathic arthritis classified by the ILAR criteria: HLA associations in UK patients. Rheumatology (Oxford). 2002;41:1183-9.

11. Di Paola M, Cavalieri D, Albanese D, Sordo M, Pindo M, Donati C, et al. Alteration of fecal microbiota profiles in juvenile idiopathic arthritis: associations with HLA-B27 allele and disease status. Front Microbiol. 2016;7:1703.

12. Kunjir V, Venugopalan A, Chopra A. Profile of Indian patients with juvenile onset chronic inflammatory joint disease using the ILAR classification criteria for JIA: a community-based cohort study. J Rheumatol. 2010;37: 1756-62.

13. Beukelman T, Patkar NM, Saag KG, Tolleson-Rinehart S, Cron RQ, DeWitt EM, et al. 2011 American College of Rheumatology recommendations for the treatment of juvenile idiopathic arthritis: initiation and safety monitoring of therapeutic agents for the treatment of arthritis and systemic features. Arthritis Care Res (Hoboken). 2011;63:465-82.

14. Silva-Ramirez B, Vargas-Alarcon G, Granados J, Burgos-Vargas R. HLA antigens and juvenile onset spondyloarthritides: negative association with non-B27 alleles. Clin Exp Rheumatol. 2005;23:721-3.

15. Khan MA, Kushner I, Braun WE. Comparison of clinical features in HLA-B27 positive and negative patients with ankylosing spondylitis. Arthritis Rheum. 1977;20:909-12.

16. Weiss PF, Xiao R, Biko DM, Chauvin NA. Assessment of sacroiliitis at diagnosis of juvenile spondyloarthritis by radiography, magnetic resonance imaging, and clinical examination. Arthritis Care Res (Hoboken). 2016;68: 187-94.

17. Berntson L, Damgard M, Andersson-Gare B, Herlin T, Nielsen S, Nordal E, et al. HLA-B27 predicts a more extended disease with increasing age at onset in boys with juvenile idiopathic arthritis. J Rheumatol. 2008;35: 2055-61.

18. Gare BA, Fasth A. The natural history of juvenile chronic arthritis: a population based cohort study. I. Onset and disease process. J Rheumatol. 1995;22:295-307.

19. Berntson L, Nordal E, Aalto K, Peltoniemi S, Herlin T, Zak M, et al. HLA-B27 predicts a more chronic disease course in an 8-year followup cohort of patients with juvenile idiopathic arthritis. J Rheumatol. 2013;40:725-31.

20. Giannini EH, Ruperto N, Ravelli A, Lovell DJ, Felson DT, Martini A. Preliminary definition of improvement in juvenile arthritis. Arthritis Rheum. 1997:40: 1202-9.

21. Brewer Jr EJ, Bass J, Baum J, Cassidy JT, Fink C, Jacobs J, et al. JRA Criteria Subcommittee of the Diagnostic and Therapeutic Criteria Committee of the American Rheumatism Section of The Arthritis Foundation. Current proposed revision of JRA criteria. Arthritis Rheum. 1977;20(2 Suppl):195-9.

22. Weiss PF, Xiao R, Biko DM, Johnson AM, Chauvin NA. Detection of inflammatory sacroiliitis in children with magnetic resonance imaging: is gadolinium contrast enhancement necessary? Arthritis Rheumatol. 2015;67: 2250-6.

23. Sieper J, Rudwaleit M, Baraliakos X, Brandt J, Braun J, Burgos-Vargas R, et al. The Assessment of SpondyloArthritis international Society (ASAS) handbook: a guide to assess spondyloarthritis. Ann Rheum Dis. 2009;68 Suppl 2:ii1-44.

24. Tennant A, Kearns S, Turner F, Wyatt S, Haigh R, Chamberlain MA. Measuring the function of children with juvenile arthritis. Rheumatology (Oxford). 2001:40:1274-8.

25. Lam C, Young N, Marwaha J, McLimont M, Feldman BM. Revised versions of the Childhood Health Assessment Questionnaire (CHAQ) are more sensitive and suffer less from a ceiling effect. Arthritis Rheum. 2004;51:881-9.

26. Dempster H, Porepa M, Young N, Feldman BM. The clinical meaning of functional outcome scores in children with juvenile arthritis. Arthritis Rheum. 2001:44:1768-74.

27. Consolaro A, Ruperto N, Bazso A, Pistorio A, Magni-Manzoni S, Filocamo G, et al. Development and validation of a composite disease activity score for juvenile idiopathic arthritis. Arthritis Rheum. 2009;61:658-66.

28. Consolaro A, Giancane G, Schiappapietra B, Davì S, Calandra S, Lanni S, et al. Clinical outcome measures in juvenile idiopathic arthritis. Pediatr Rheumatol Online J. 2016:14:23.

29. McErlane F, Beresford MW, Baildam EM, Chieng SE, Davidson JE, Foster HE, et al. Validity of a three-variable Juvenile Arthritis Disease Activity Score in children with new-onset juvenile idiopathic arthritis. Ann Rheum Dis. 2013; 72:1983-8.

30. Consolaro A, Negro G, Chiara Gallo M, Bracciolini G, Ferrari C, Schiappapietra $B$, et al. Defining criteria for disease activity states in nonsystemic juvenile idiopathic arthritis based on a three-variable juvenile arthritis disease activity score. Arthritis Care Res (Hoboken). 2014;66:1703-9.

31. Weiss PF, Colbert RA, Xiao R, Feudtner C, Beukelman T, DeWitt EM, et al. Development and retrospective validation of the Juvenile Spondyloarthritis Disease Activity Index. Arthritis Care Res (Hoboken). 2014;66:1775-82.

32. Burgos-Vargas R. The juvenile-onset spondyloarthritides. Rheum Dis Clin North Am. 2002;28:531-60.

33. Katsicas MM, Russo R. Biologic agents in juvenile spondyloarthropathies. Pediatr Rheumatol Online J. 2016;14:17.

34. Homeff G, Burgos-Vargas R. TNF-a antagonists for the treatment of juvenileonset spondyloarthritides. Clin Exp Rheumatol. 2002;20(6 Suppl 28): S137-42. 
35. Tse S, Burgos-Vargas R, Colbert RA. Juvenile spondyloarthritis treatment recommendations. Am J Med Sci. 2012;343:367-70.

36. Zeboulon N, Dougados M, Gossec L. Prevalence and characteristics of uveitis in the spondyloarthropathies: a systematic literature review. Ann Rheum Dis. 2008;67:955-9.

37. Stoll ML, Bhore R, Dempsey-Robertson M, Punaro M. Spondyloarthritis in a pediatric population: risk factors for sacroiliitis. J Rheumatol. 2010;37: 2402-8.

38. Bollow M, Biedermann T, Kannenberg J, Paris S, Schauer-Petrowski C, Minden $\mathrm{K}$, et al. Use of dynamic magnetic resonance imaging to detect sacroiliitis in HLA-B27 positive and negative children with juvenile arthritides. J Rheumatol. 1998;25:556-64.

Submit your next manuscript to BioMed Central and we will help you at every step:

- We accept pre-submission inquiries

- Our selector tool helps you to find the most relevant journal

- We provide round the clock customer support

- Convenient online submission

- Thorough peer review

- Inclusion in PubMed and all major indexing services

- Maximum visibility for your research

Submit your manuscript at www.biomedcentral.com/submit
Biomed Central 